\title{
Next Generation Vehicle Diagnostics
}

\author{
Dr. Jörg Supke \\ EMOTIVE GmbH \& Co. KG, Pfingstweideweg 17, D-73760 Ostfildern, Germany, www.emotive.de \\ contact@emotive.de
}

\begin{abstract}
:
The next generation vehicle diagnostics have to master the ever-increasing complexity in automotive electronics. Due to its integrative and harmonizing effects, the new OTX standard plays a key role for this challenge. As a meta-language, OTX can bring together different, previously separate standards more effectively than any other standard. OTX is a platform and tester independent exchange format for executable test sequences. The XML-based test sequence language offers the opportunity to exchange test knowledge across departments, tools and process boundaries. The paper shows the practical experiences and benefits of cross-sectoral use of OTX at a German vehicle manufacturer in development, production, service and within the vehicle. It will demonstrate the leading role OTX plays in solving diagnostic tasks inside the new end-to-end electronic architecture [5].
\end{abstract}

Key words: test language, test knowledge, exchange format, vehicle diagnostics, OTX

\section{Introduction}

We live in a connected world. The worldwide availability of a secure broadband and crosssystem communication, the increasingly demanding customer requirements and the ever-growing global competition result in a permanent pressure on all operational procedures and processes. The well-known trends in automotive electronics

- Onboard diagnostic tester,

- Onboard high-performance computer,

- Onboard gigabit ethernet,
- Standardized webservice interface for access to the vehicles,

- Service-oriented architecture replaces component-oriented architecture,

- Cloud-based outsourcing of services,

- Future integration of functions currently unknown

as well as the resulting complexity (see Fig. 1), challenges companies to continually improve their operations. The degree of the "relaxed" mastery of complexity makes the quality of operational processes visible.

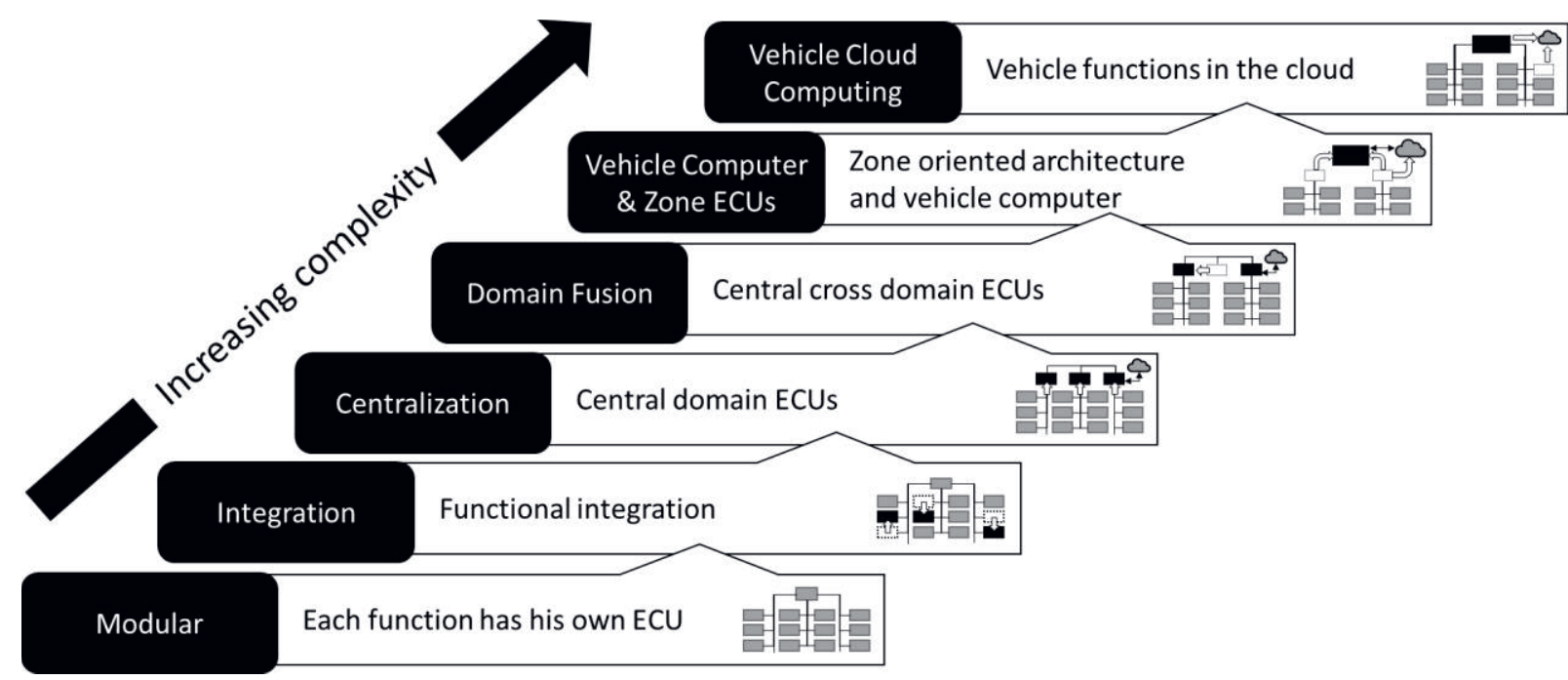

Fig. 1. Evolution of E / E architecture 
So-called end-to-end processes have become a leading principle. An end-to-end process is a process that consists of all consecutive subprocesses that are necessary to fulfill a specific customer need. The emphasis on end-to-end is to make people aware that this process extends from the needs of the client to the delivery of services. This process is typically crossdepartmental. A holistic end-to-end process organization is therefore the key to operational excellence [4].

Standards play a key role in this development. They establish uniform fundamentals for the exchange of components worldwide. They are based on provable scientific arguments and target macroeconomic purposes. The benefit for all is beyond the benefit of individuals or institutions. Basically, a standard is a recommendation and the use is optional. Because of the significant importance for the interplay of technical and economic solutions, the widest possible acceptance and application of standards is necessary and useful.
The cross-linking of the vehicle and the resulting technological leap offers the opportunity to establish consistent and standardized end-to-end processes. In this evolution standardization should be promoted and existing standards should be strengthened. This is a requirement for the continuous improvement, the consistency of value generation and the avoidance of redundancies.

\section{OTX Standard}

Due to its integrative and harmonizing effect, the OTX standard is of particular importance. OTX stands for Open Test sequence eXchange and it is standardized in ISO 13209. As a metalanguage, OTX seamlessly integrates with existing diagnostic communication standards such as ODX and $\mathrm{MVCl}$ (see Fig. 2). Like no other standard OTX can bring together previously separate standards such as ASAM GDI, ASAM XIL or MCD3-MC. With OTX test knowledge can be exchanged between different departments in development, production and after-sales.

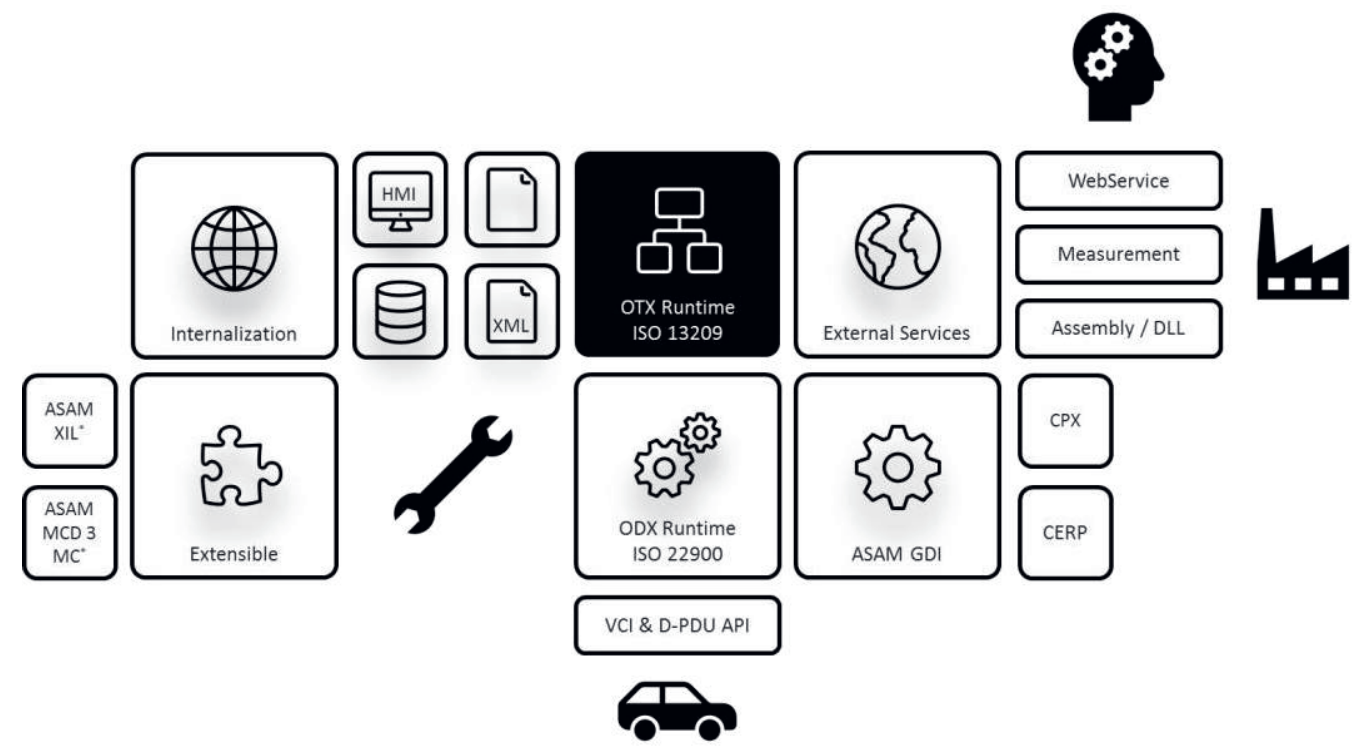

Fig. 2. OTX can connect standards and applications in other areas

The target of OTX is the process-reliable exchange, archiving and execution of test knowledge. With the support of appropriate software tools, it makes the diagnostic development process easier and more productive. OTX describe executable test logic with testable quality. OTX is machine and human readable. It stores test knowledge and is independent of the technology, the service provider and the tool manufacturer. OTX is open and stable. OTX has a strict separation of test logic and runtime implementation. Therefore, OTX is platform independent. OTX is actively developed within the ASAM and the ISO/DIN and has a broad tool support.

An OTX sequence consists of one or more activities. All activities are thematically grouped in OTX libraries so called OTX Extensions. The OTX core library [1] contains all the activities for the general test logic, such as procedure calls, assignments, branches, loops, activities for parallel execution, error handling and furthermore. All 36 extensions (see [2] and [3]) extend the stand-alone executable core by specific functionalities (see Fig. 3). Besides extensions for vehicle diagnostics, the HMI or 
the access to an arbitrary external system via the "External Service Provider" extension, there are a lot of extensions, which can cover almost every aspect of testing in the automotive industry. Furthermore, OTX can be expanded easily and standard compliant.

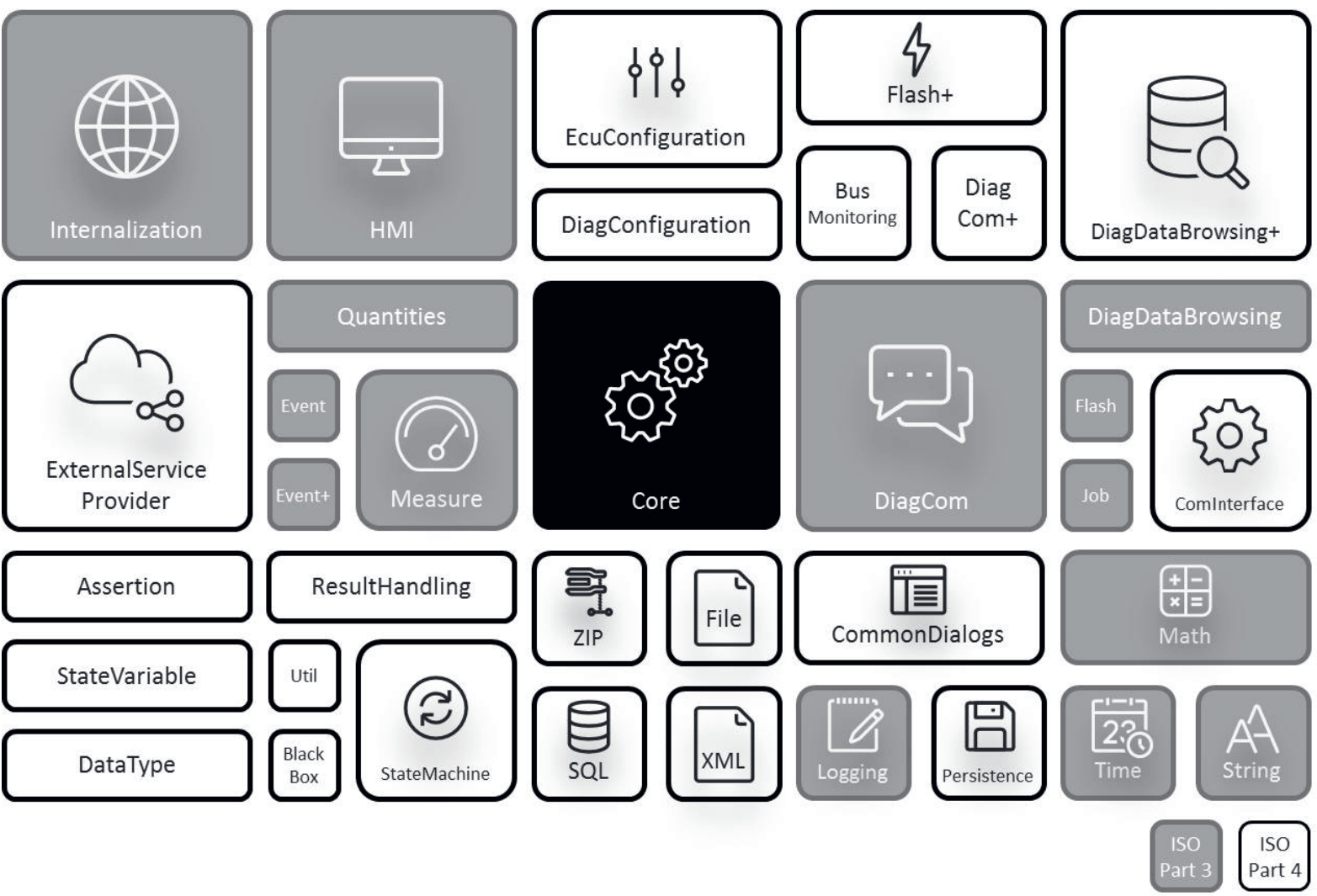

Fig. 3. Overview of the latest 36 standardized OTX Extensions

OTX is stored in XML documents. The following picture shows a very simple example of an OTX sequence (see Fig. 4). Within the procedure named "main", the value "Hello World" is assigned to "Variable1". The same procedure is shown on the right as OTL. OTL is a Java-like scripting language based at the OTX data model. In the EMOTIVE development environment Open Test Framework (see Fig. 9), an author can develop the sequences in a graphical workflow designer or in the OTL Code Editor. Designer and Code Editor synchronize themselves continuously.
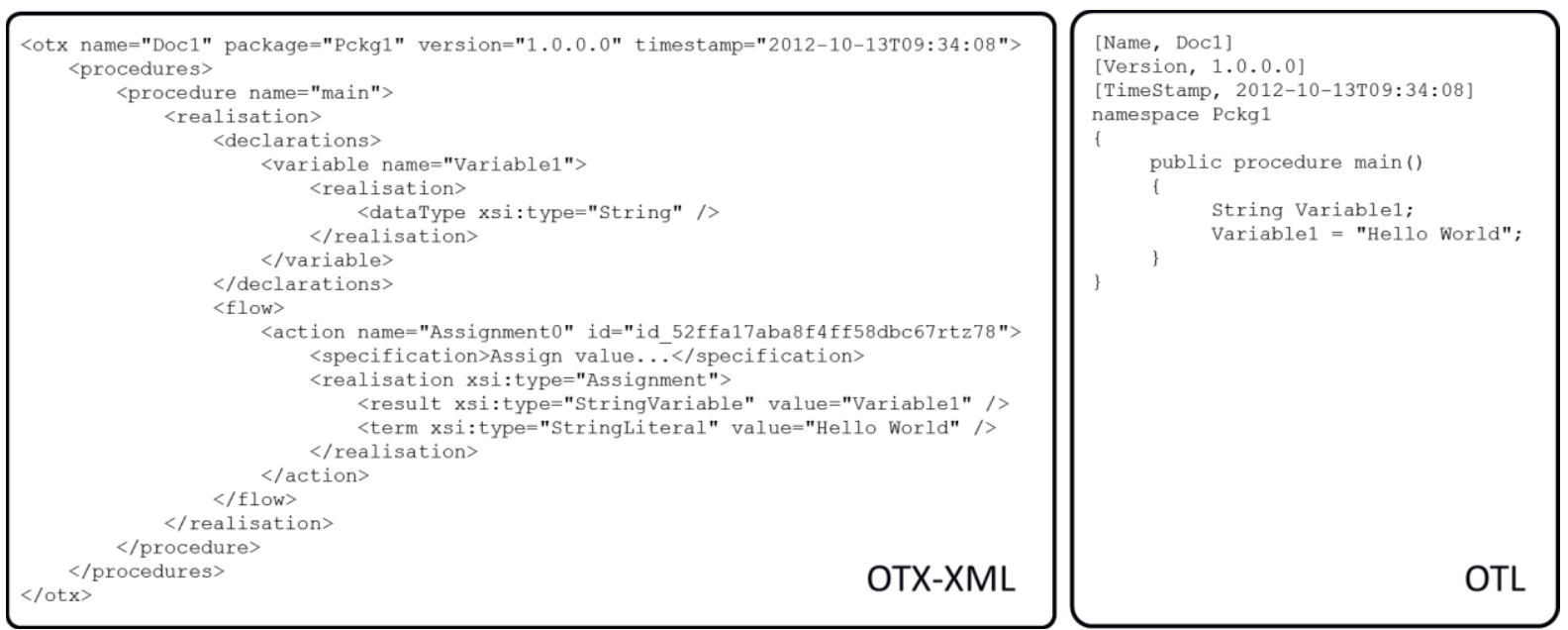

Fig. 4. OTX "Hello World" sample in XML and OTL 


\section{Access to external Systems - OTX Mapping}

One of the biggest advantage of OTX is the ability to separate test logic and runtime implementation. This allows an access to any external system in the environment. Together with a German vehicle manufacture, EMOTIVE has developed the so-called OTX Mapping. The aim was, that simply by exchanging a mapping file, the same OTX sequence should be executed in different environments. Because of that, the OEM was able to use the same OTX sequence without changing the test logic in development, production, after-sales and inside the vehicle. This significantly reduces the effort required to create, edit, validate and maintain the test logic. It reduces the dependency on suppliers and by the way a uniform, standardized documentation without additional effort is available.

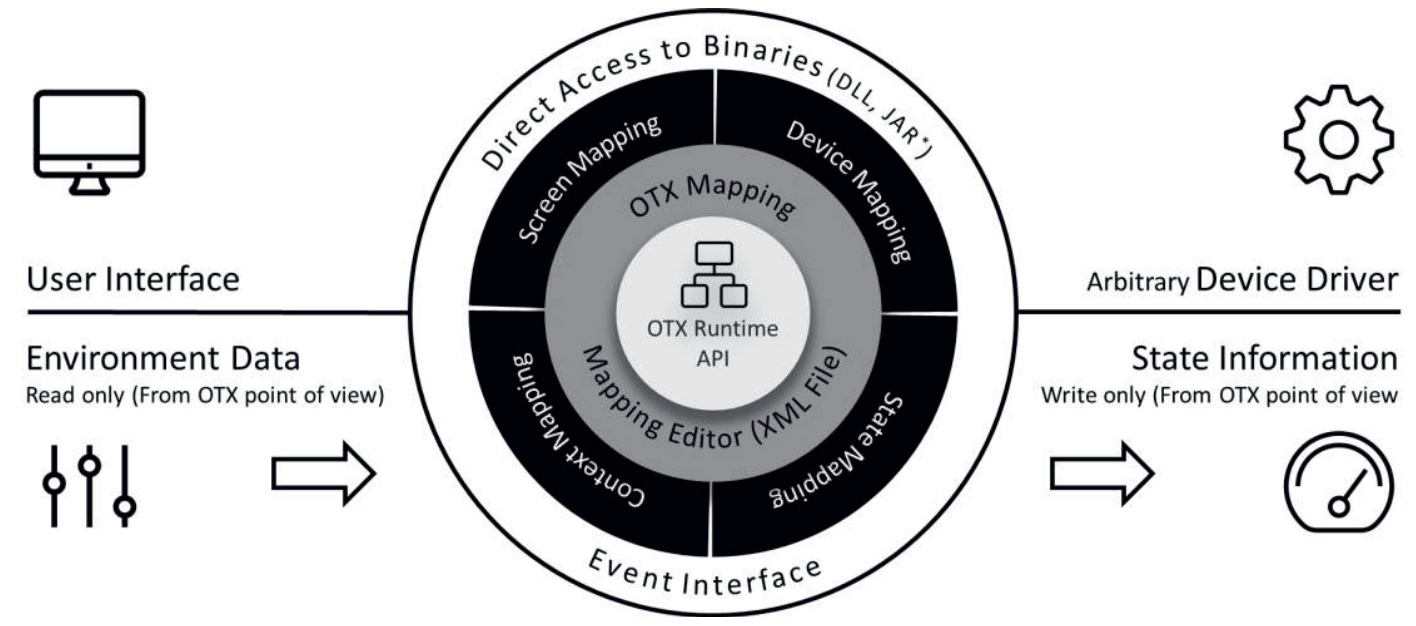

Fig. 5. Access to external systems - OTX Mapping

The OTX Mapping consists of four parts (see Fig. 5): The Screen Mapping for the interaction with any user interface. With the Screen Mapping the parameters of a so-called OTX "Screen Signature" are bound to the properties of GUI controls. With the Device Mapping, any function in an external system can be called via a so-called OTX "Device Service Signature", such as reading the terminal-15 state. Via the Context Mapping values from the environment can be read and via the State Mapping status information into the environment can be transferred.

The following simplified example for the calibration of tire pressure sensors should illustrate the OTX Mapping for different target systems (see Fig. 6).

At first the ID of a tire pressure sensor must be entered for three different environments via the Context Mapping. Inside the production the ID will automatically entered via the test system. Within the development it will entered via a barcode scanner and for a service station it will entered manually. At next the entered value must be validated. After then a routine will be started via a diagnostic service to calibrate the sensors. The routine will be monitored and parallel the calibration process will be visualized in three different ways via the Screen Mapping:
Within the production via a screen inside the test system. In the development via a simple generic screen and in the after-sales via a screen inside the infotainment system of the car. The last two steps calculate, log and print out the result.

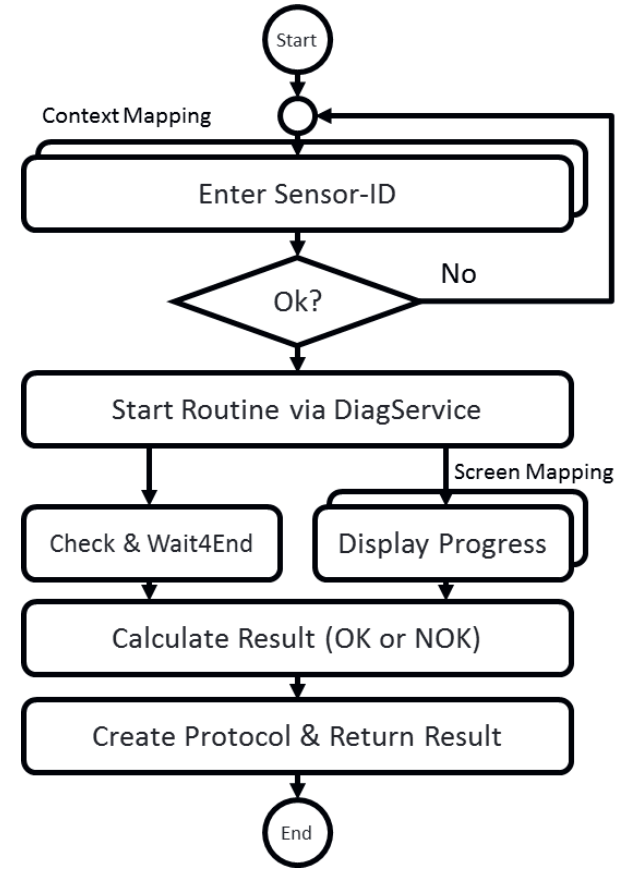

Fig. 6. Use case example - tire pressure sensor calibration 
Fig. 7 shows the process for developing this setup sequence. It starts with an abstract specification of the simple test logic like in Microsoft Visio. The result is an initial OTX document. In the following implementation phase, all specifications will be implemented using an OTX test step library. A completely executable OTX document will be generated and tested in the next step. The result is a standardized PTX file, which can be transferred to other departments. Each department adds its environment-specific OTX Mapping and creates a so-called PPX file. While a PTX file is platform-neutral, a PPX file contains platformspecific information in the form of the OTX Mapping file. The PPX file can be executed on different target systems. In application 1, the sequence is executed within an existing system in production or after-sales. Application 2 executes the same PPX within a generic pilot tester within the prototype workshop of the development.

In summary, it can be said that with the new OTX Mapping it is possible to exchange test knowledge through adaptation and enrichment across departments in an efficient way never seen before. A test sequence is specified, implemented and tested in the development. It is adapted and enriched for production, aftersales and for the use inside the vehicle without changing the test logic.

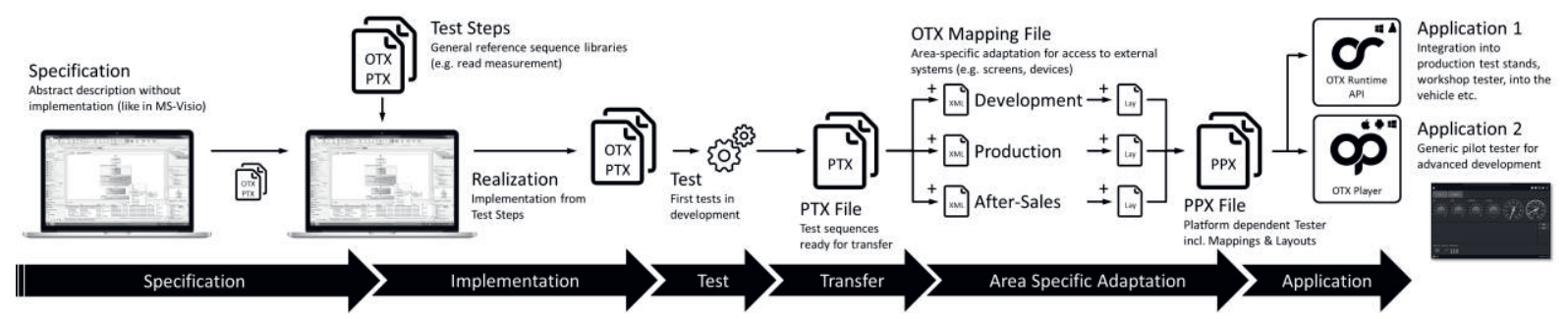

Fig. 7. Development of a start-up sequence step by step

\section{OTX Tool Chain}

Due to the standardization process and the resulting possibilities, a complete OTX tool chain was developed by EMOTIVE (see Fig. 8).

The tool chain is in productive use at several OEMs in development, production, after-sales and inside the vehicle. It mainly consists of the OTX development environment "Open Test Framework", the OTX Runtime API and the OTX Player.
The Open Test Framework (OTF) is a complete development environment for designing, maintaining, and testing a new generation of process-reliable tester applications that run on Windows and Linux (see Fig. 9). The OTF has a clear and intuitive user interface. It helps sequence designers and project managers to create and deliver tester solutions faster and without any programming skills. All relevant standards such as ISO 13209 (OTX), ISO 22900 (MVCI) and ISO 22901 (ODX) are supported.

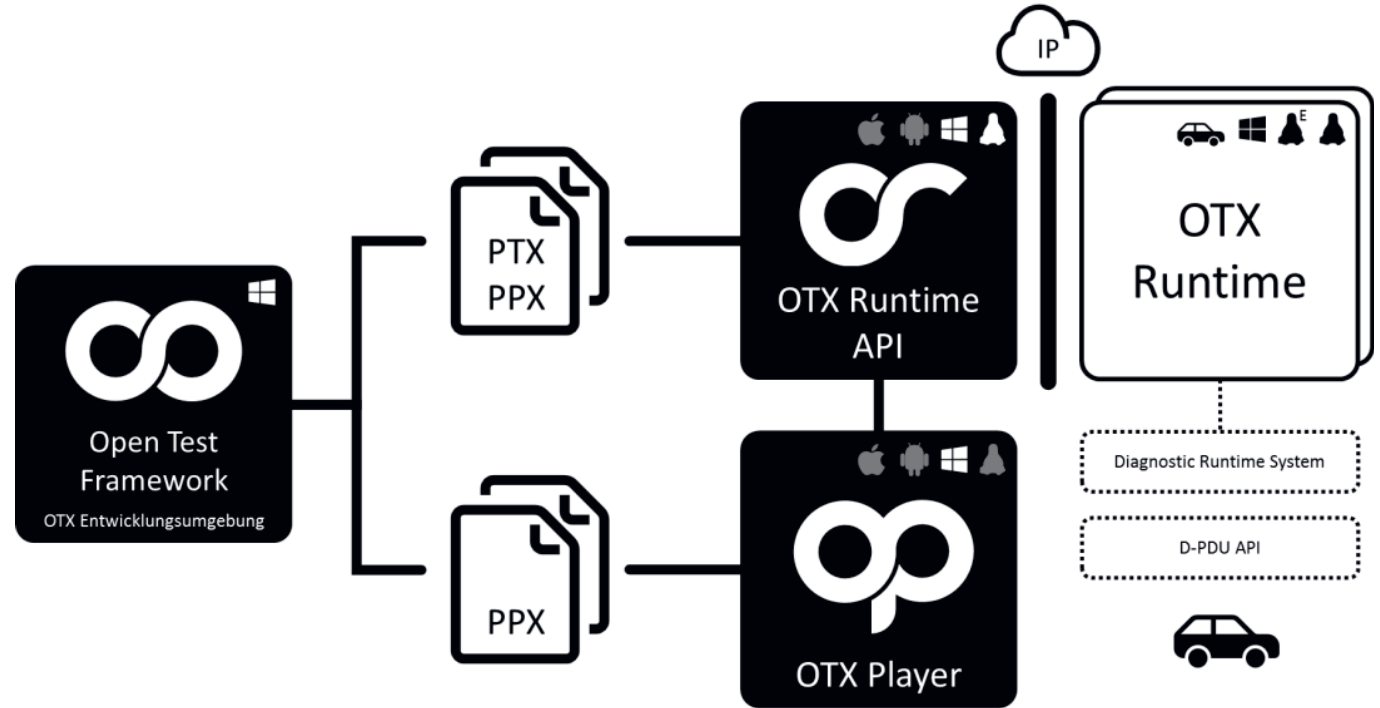

Fig. 8. OTX tool chain of EMOTIVE 
The areas of application of the OTF are the basic work with standardized test sequences, the creation of redistributable players etc. Applications inside off-board vehicle diagnostics are in the foreground. Examples are a universal and quickly adaptable Flash tool, a tool for checking the ECU protocol conformity, simple and complex development and production testers or even a simple scan tool in the OBD environment.

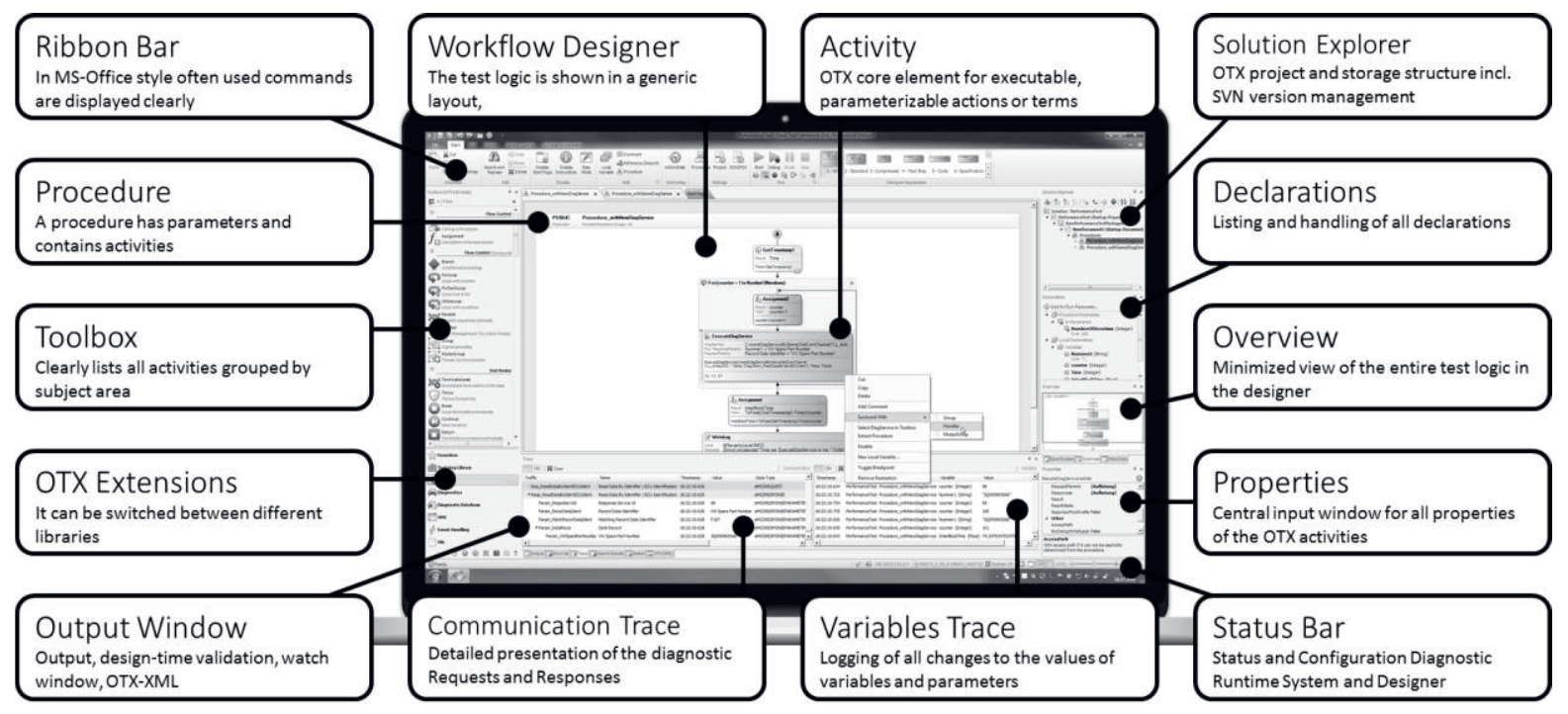

Fig. 9. OTX development environment Open Test Framework

The Open Test Runtime API (OTR) is a programming interface for the simple execution of OTX procedures in own applications. It works independently even without the development environment and is available for Windows, Linux and Embedded Linux. It has comprehensive possibilities for seamless integration into existing systems and infrastructures (see Fig. 10). The key features of the OTX Runtime API are:

- Enables users to use OTX in their own applications

- API for Java, DotNet and COM

- Runs on Windows, Linux and Embedded Linux

- Synchronous and asynchronous execution of OTX sequences

- Multi-threading and multi-instance capable

- Long-term stable and performant

- Remote capable

The application areas of the Open Test Runtime API range from the simple execution of
PTX files through the integration in test benches of different manufacturers to the complete and seamless integration of OTX into own applications.
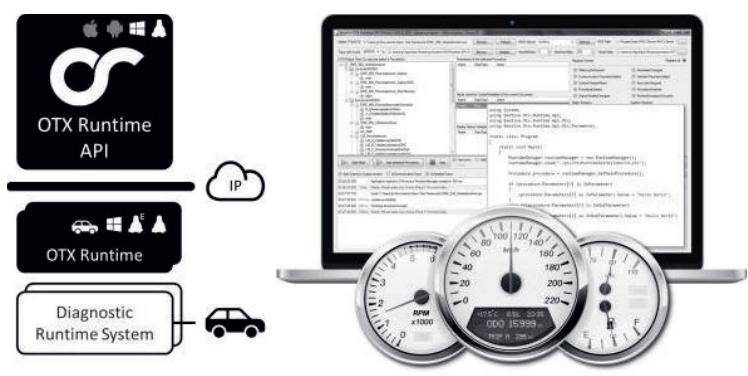

Fig. 10. Open Test Runtime API (OTX runtime) for seamless integration into existing systems

The Open Test Player (OTP) is a complete generic, OTX based tester for the simplest distribution and execution of test sequences (see Fig. 11). It is completely based on the Open Test Runtime API and can be easily adapted to the company look and feel. It works with Windows. Implementations for iOS and Android are planned. A player project (PPX) can be graphically created and tested in the Open Test Framework without any programming skills. 


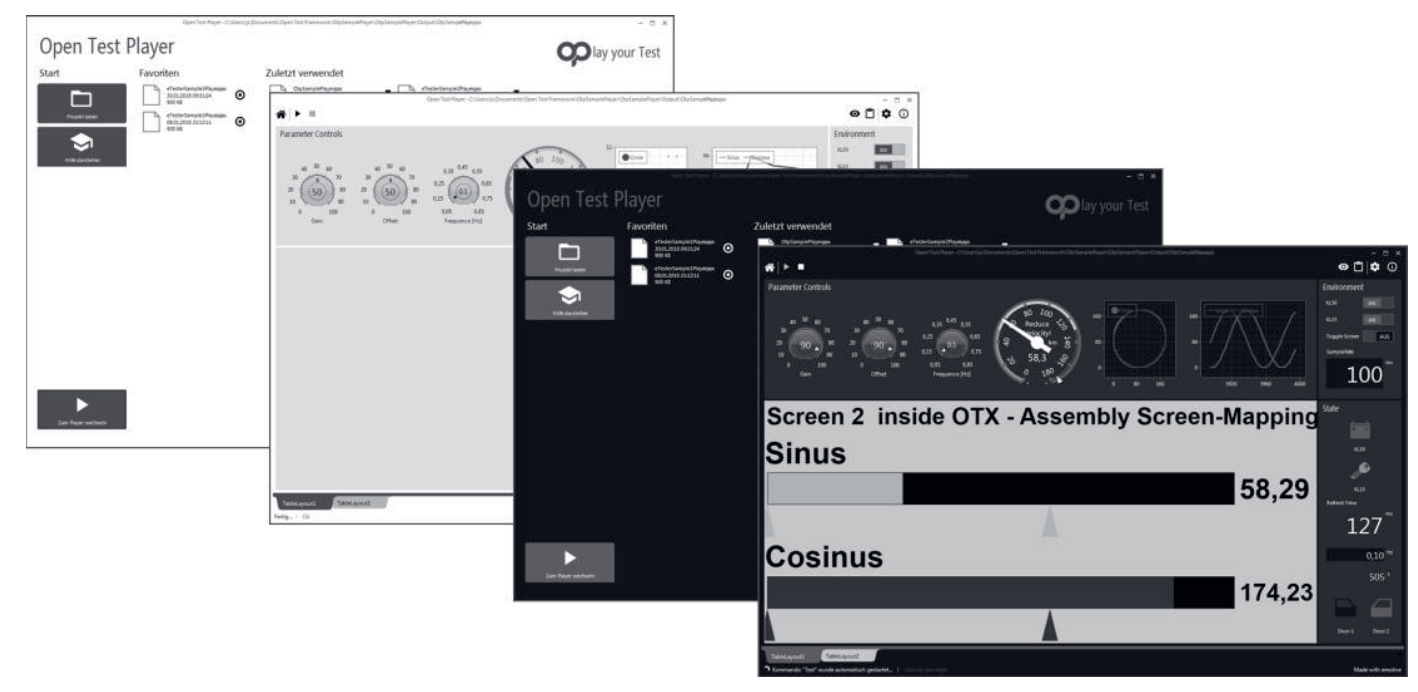

Fig. 11. Open Test Player with theme support

The key features of the OTP are:

- $100 \%$ generic OTX based tester

- For the simplest distribution and execution of test sequences

- Modern interface with company $\mathrm{Cl}$

- Based completely on the OTX Runtime API

- A player project (PPX) is created in the OTF without programming skills

- Install player, load PPX, run ... Done!

Due to the easiness of development of a player without programming knowledge, the OTP is used especially when established testers, which cannot be adapted quickly and flexibly enough for new ECUs or test sequences. For example, in the prototype workshop of development and production: Together with the OTP, ECUs can be setup quickly and easily without the use of numerous special tools. Component owners can also use the Open Test Player to deliver their ECU together with a player project that provides the basic functions of the ECU.

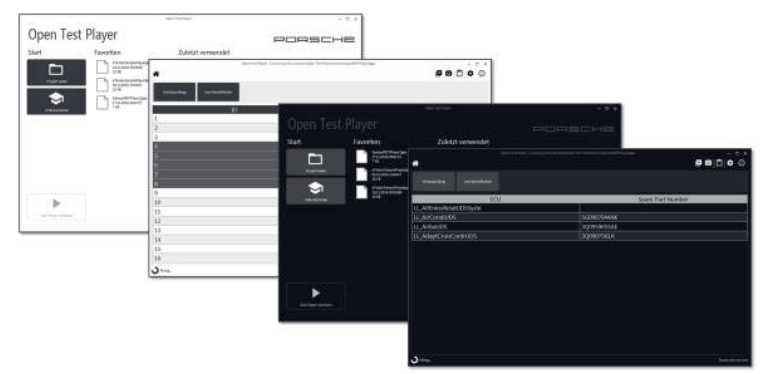

Fig. 12. Open Test Player with company Cl

The design of the Open Test Player can easily be changed via themes (see Fig. 12). The user can adapt the logo, the colors, the fonts and the icons inside the Theme Manager of the Open Test Framework. A theme file with the company $\mathrm{Cl}$ can be created, transferred and loaded into the OTP.

\section{Summary}

The new standard OTX (Open Test sequence eXchange) is an executable, platform and tester independent exchange format for test sequences, standardized in ISO 13029. It leads to a substantial simplification of the processes to create and maintain standardized test logic. OTX enables the reliable archiving of testing knowledge, it can bring together different standards and promotes integrated end-to-end processes. This next generation of vehicle diagnostics significantly reduces time and cost to master the increasing complexity.

\section{References}

[1] ISO 13209-2:2011, Road vehicles — Open Test sequence eXchange format (OTX) - Part 2:

Core data model specification and requirements, 2011

[2] ISO 13209-3:2012, Road vehicles — Open Test sequence eXchange format (OTX) - Part 3: Standard extensions and requirements, 2012

[3] ASAM Base Standard OTX Extensions, Open Test eXchange format, Interface Definition, Version 2.0.0, 2017

[4] Operational excellence, Wikipedia, https://www.wikipedia.org/wiki/Operational_excell ence

[5] Dr. Jörg Supke, Next Generation Vehicle Diagnostics (German), Diagnose in mechatronischen Systemen XII, Thelem Universitätsverlag, 2018 